\section{Fading techniques and concept learning in children*}

\author{
STEVEN H. SCHWARTZ and IRA J. FIRESTONE \\ Wayne State University, Detroit, Mich. 48202 \\ and \\ STEPHEN TERRY \\ University of Connecticut, Storrs, Conn. 06268
}

The utility of stimulus fading as a training technique for attainment of concepts was questioned. Fading on a cue which focused attention toward the criterial aspect of the stimuli (axis of symmetry) produced no better performance than standard discrimination procedures, while fading on an extrinsic dimension yielded significantly poorer performance than did the standard. Possible explanations were discussed in terms of "blocking" effects.

Stimulus fading has been proposed (Terrace, 1963, 1966) as a training procedure that can facilitate the learning of difficult discriminations. The present study was designed to determine if this type of training can facilitate the acquisition of discriminations of a conceptual character-those where an invariant difference must be abstracted from sets of perceptually heterogeneous stimuli. The importance of such a demonstration can readily be seen in that the benchmarks of cognitive development (e.g., Piaget's conservation) require perception of high order or relational invariances embedded in stimuli of variable physical configuration.

Fading involves the gradual shifting of stimulus control from some dominant stimulus dimension (e.g., brightness) to a desired terminal discrimination on a dimension less salient for the organism (e.g., angle of orientation). The process of acquisition under such fading procedures is often described as "errorless" (Leith \& Haude, 1969; Terrace, 1963), as there is little responding to incorrect nonreinforced stimuli. Furthermore, difficult discriminations and those seemingly unattainable by conventional procedures have been achieved with fading. Moore \& Goldiamond (1964) were able to train preschoolers to find matches for sample stimuli (triangles) from an array of comparison stimuli in different degrees of rotation only through the fading out of an extrinsic brightness cue. Likewise, Stettner \& Matyniak (1969) achieved a pattern discrimination (vertical-horizontal) in a brain-damaged quail by fading out a superimposed difference in hue after no success on over 2,000 trials of standard training.

Fading procedures are not, however,

* Supported in part by PHS Grant MH 17723-01 to the first author. universally successful. A number of researchers (Gollin \& Savoy, 1968; Schusterman, 1966; Sidman \& interdimensional transfer of stimulus control may result in errors or even complete failure if not done in a "proper" or gradual enough fashion. For example, McCleave (1965) reports failure to teach a preschool child to discriminate a backward " $C$ "' from a proper " $C$," using the size of the letters as the fading dimension. A noticeable size difference was sufficient to allow errorless responding; but chance responding reappeared when the backward " $C$ " was faded to the same size as the other "C." Learning was ultimately achieved here on the basis of an available discrimination between open and closed letter forms; the " $C$ " was paired with an " $O$ " that gradually faded open to the backward "C."

The sensitivity of even such a simple invariant element discrimination problem to mode of fading approach suggests the importance of attentional processes as mechanisms underlying interdimensional transfer of stimulus control. Fading on dimensions which explicitly focus the S's attention toward the required higher-order invariances should be more efficacious than fading on dimensions which serve only to make the initial discriminations easy and therefore errorless. To test this hypothesis, we compared two broad types of fading procedures, one where the fading dimension has no particular relation to the concept (extrinsic fading) and a second where the fading dimension focuses attention toward the conceptual classification variable (intrinsic fading). Since the intrinsic fading cues should draw attention to the invariant differences between successive sets of instances and noninstances of the concept, while the extrinsic fading cues may distract from these differences, superior performance is expected in the former Stoddard, 1967) have found that compared to the latter condition. The magnitude and direction of each of these effects is assessed in terms of the control group given traditional discrimination training.

\section{METHOD}

Bilateral symmetry was selected as the task, since it typifies a class of concepts wherein the distinction between exemplars and nonexemplars are based on abstract relational properties rather than absolute dimensions or elements. Numerous and diverse instances of this class can be readily produced, and it is apparently the case that most young children do not have such a concept prior to explicit training (St. Claire, 1968).

The sample of stimuli defining the concept consisted of 16 pairs of fiveand six-sided figures. Each pair involved one symmetric and a rather similar asymmetric drawing (Fig. 1). These pairs of figures appeared in three orientations: once each with the axis of symmetry in the vertical, horizontal, and diagonal (45-deg) planes. The inclination of the asymmetric figure was governed by that of the symmetric item; thus, if the point of the five-sided figure (right, Fig. 1) was rotated 45 deg from the vertical, the corresponding point on the asymmetric paired item was also set at $45 \mathrm{deg}$. It is recognized that rotation from the vertical makes the task more difficult. However, we considered this necessary to be certain that the child had achieved the concept and not specific shape recognition.

A saturation difference served as the prompting cue. In extrinsic fading (Fig. 1a), saturation was gradually increased on the entire area of the symmetric member of the stimulus pair as training progressed. Thus, the symmetric member gradually approached the constant $100 \%$ saturation value of the asymmetric member. Intrinsic fading (Fig. 1b) differed only in that the saturation fading was applied to half of the

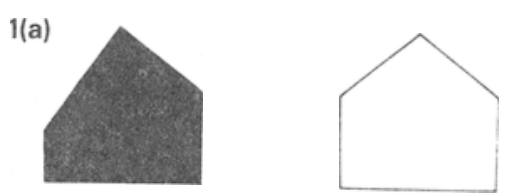

1 (b)

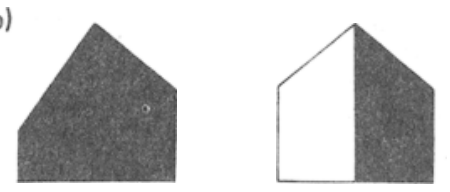

Fig. 1. Fading stimuli (sample): (1a) extrinsic; (1b) intrinsic. 
Table 1

Performance Measures on Symmetry Concept in Three Treatment Conditions (N $=20$ Per Condition)

\begin{tabular}{|c|c|c|c|c|c|}
\hline & & & \multicolumn{3}{|c|}{ Treatment Condition } \\
\hline \multicolumn{2}{|r|}{ Variable } & & $\begin{array}{l}\text { Extrinsic } \\
\text { Fading }\end{array}$ & $\begin{array}{c}\text { Intrinsic } \\
\text { Fading }\end{array}$ & $\begin{array}{r}\text { Standard } \\
\text { Control } \\
\end{array}$ \\
\hline 1. & $\begin{array}{l}\text { Proportion of } \mathrm{Ss} \\
\text { attaining concept }\end{array}$ & & $.20 * *$ & .60 & .70 \\
\hline 2. & Number of errors & $\begin{array}{l}\overline{\mathbf{X}} \\
\sigma\end{array}$ & $\begin{array}{c}12.30^{*} \\
6.02\end{array}$ & $\begin{array}{l}8.25 \\
4.18\end{array}$ & $\begin{array}{l}8.80 \\
4.32\end{array}$ \\
\hline 3. & $\begin{array}{l}\text { Proportion errors } \\
\text { at Level } 6 \text { (no } \\
\text { prompting cues) }\end{array}$ & $\begin{array}{l}\overline{\mathrm{X}} \\
\sigma\end{array}$ & $\begin{array}{l}.36 * * \\
.21\end{array}$ & $\begin{array}{l}.20 \\
.18\end{array}$ & $\begin{array}{l}.18 \\
.15\end{array}$ \\
\hline 4. & $\begin{array}{l}\text { Proportion errors at } \\
\text { Levels } 1-5 \text { (prompt- } \\
\text { ing cues present) }\end{array}$ & $\begin{array}{l}\overrightarrow{\mathrm{X}} \\
\sigma\end{array}$ & $\begin{array}{l}.06 \\
.03\end{array}$ & $\begin{array}{l}.05 \\
.03\end{array}$ & -+ \\
\hline
\end{tabular}

tNo prompting cues ever present in this condition

${ }^{*} p<.05$ (compared to control group)

$* * p<.01$ (compared to control group)

symmetric member. Thus, this member initially appeared two-toned around its axis of symmetry. As training progressed, the saturation of the less dense half increased to $100 \%$, resulting in a figure of uniform saturation. For both fading groups, a correct response reduced the saturation difference on the next stimulus pair by one step, while an incorrect response increased this difference equivalently. Six levels of color (brown) saturation $(0 \%, 10 \%$, $30 \%, 50 \%$, $70 \%$, and $100 \%)$ were available on each figure for both fading series. In the standard discrimination training, all figures were of equal (100\%) saturation throughout. All Ss responded to 48 stimuli (16 figures in three orientations). Ten consecutive correct responses in the absence of a prompting cue served as the criterion for attainment of the concept.

The experiment was conducted in an integrated, largely middle-class, urban elementary school. Sixty first-grade children were assigned randomly to the three treatment conditions to a limit of 20 per condition. The task was introduced as a game wherein marbles (exchangeable for prizes) would be won by "picking the correct picture from each set of two." Stimuli were presented in a back-lit box containing two $3 \times 3$ in. windows, 3 in. apart, on the front surface. A button placed below each window allowed $S$ to indicate his choice. Correct responses activated a marble dispenser and (following a 2 -sec delay) a shutter covering the windows. Position preferences were weakened in a short series of practice trials which familiarized the Ss with the color saturation cue.

\section{RESULTS AND DISCUSSION}

Overall performance data are summarized in Table 1. As expected, performance under extrinsic fading was poorer than under the standard concept attainment paradigm. Only $20 \%$ of the extrinsic-fading Ss attained the symmetry concept compared to $70 \%$ of the control Ss $\left[x^{2}(1)=10.1\right.$, $p<.01]$. However, contrary to predictions, the intrinsic fading produced no better performance than the control training (60\% attainment, $x^{2}<1$ ). Number of errors made during training (Variable 2) reveal similar differences, with the extrinsic group performing worse than the controls $[t(38)=2.06, p<.05]$, and no significant differences between intrinsic and control groups $(t<1)$.

The locus of the poor performance of the extrinsic-fading Ss does not lie in their inability to utilize the prompting cue. For trials on which the fading prompt was available, their error rate (Measure 4) was only .06, compared to .05 for the intrinsic group $(t<1)$. Rather, their difficulty occurred at Level 6, where the prompting cue had been completely faded out. Here (Measure 3 ) the extrinsic Ss commit errors on .36 of the trials compared to .20 and .18 for the other conditions.

Inferior performance under extrinsic fading is consistent with findings obtained on relevant redundant cue problems (Trabasso \& Bower, 1968). They report a general "blocking" or "overshadowing" effect, wherein a first-learned cue "blocks" the learning of subsequently presented redundant cues. Even when two relevant redundant cues are provided at the onset of training, the overwhelming majority of Ss learn only one. Our fading cue (saturation difference) is likely to be the one initially attended to, since it was deliberately chosen for its saliency. The low error rates obtained on trials where this cue is present (Measure 4) support this contention. By the time the child learns that the extrinsic fading cue will not always be present, he has the task of extinguishing a well-learned response to this cue and must begin searching for a new hypothesis with no particular guidance as to where to start.

The blocking effects cited by Trabasso and Bower may also explain the absence of any facilitative effect for intrinsic fading. Here again, Ss must extinguish their responses to the fading cue. However, since the fading cue in this case is more closely tied to the critical dimension, it may increase the likelihood of that dimension's being noticed. Hence, one would expect a solution rate comparable to the control condition but achieved later in the training. In fact, the mean trials to solution for solvers in the intrinsic group was 29.5 compared to 15.3 for the control group $[t(24)=2.42, p<.05]$.

In summary, our results suggest that for acquisition of conceptual discriminations, where an invariant difference must be abstracted from sets of perceptually heterogeneous stimuli, the standard discrimination paradigm is as effective as even an intrinsic fading program and more effective than an extrinsic one.

\section{REFERENCES}

GOLLIN, E. S., \& SAVOY, P. Fading procedures and conditional discrimination in children. Journal of the Experimental Analysis of Behavior, 1968, $11,443-451$.

LEITH, N. J., \& HAUDE, R. H. Errorless discrimination training in monkeys Proceedings of 77th Annual Convention, APA, 1969, 799-800.

MCCLEAVE, J. C. Applications of operant principles in teaching visua discrimination to a visually handicapped autistic child. Unpublished thesis, University of Washington, 1965.

MOORE, R.. \& GOLDIAMOND, I. Errorless establishment of visual discrimination using fading procedures. Journal of the Experimental Analysis of Behavior, 1964, 7, 269-272.

SCHUSTERMAN, R. J. Serial discrimination-reversal learning with and without errors by the California sea-lion. Journal of the Experimental Analysis of Behavior, 1966, 9, 593-600.

SIDMAN, $M$ \& STODDARD, $L$. $T$ The effectiveness of fading in programming a simultaneous form discrimination for retarded children. Journal of the Experimental Analysis of Behavior, 1967. 10, 3-15.

ST. CLAIRE, I. Z. A study of the development of the concept of symmetry by elementary school children. Unpublished dissertation, University of Texas at Austin, 1968.

STETTNER, L., \& MAYTNIAK, K. The use of stimulus fading in assessing behavioral deficits produced by brain damage. Physiology \& Behavior, 1969, 4, 859-861. TERRACE, H. S. Discrimination learning with and without "errors." Journal of the Experimental Analysis of Behavior, 1963, 6. 1-27.

TERRACE, H. S. Stimulus control. In W. Honig (Ed.), Operant behavior: Areas of research and application. New York: Appleton-Century-Crofts, 1966 . Pp. 271-344.

TRABASSO, T., \& BOWER, G. H. Attention in learning: Theory and research. New York: Wiley, 1968. 